\title{
A Mini-Review on High-Penetration Renewable Integration Into a Smarter Grid
}

\author{
Yang $L i^{*}$, Chunling Wang and Guoqing Li \\ School of Electrical Engineering, Northeast Electric Power University, Jilin, China
}

With the increasingly serious energy crisis and environmental pollution, the development and utilization of renewable energy resources have become an indispensable choice to ensure secure and sustainable energy supply. In recent years, the installed capacities of renewable generations such as wind power and photovoltaic are rapidly increasing, changing power generating sources of power systems from predominantly fossil power generation to high penetration of renewable generations. Unfortunately, the inherent uncertainty and variability of renewable energy sources will pose huge operational challenges in today's power systems. At the same time, new emerging techniques, such as smart inverters, advanced communication protocols, and machine learning, provide more regulatory means available for better integration of high-penetration renewables. This paper deals with a mini-review of several emerging technologies for high-penetration renewable integration (HPRI). The main aim of this paper is the introduction of the

OPEN ACCESS

Edited by:

Shuqing Zhang,

Tsinghua University, China

Reviewed by:

Haibo $L i$,

Tsinghua University, China

Fengqiu $\mathrm{Xu}$

Wuhan University, China

*Correspondence:

Yang Li

liyang@neepu.edu.cn

Specialty section: This article was submitted to Smart Grids,

a section of the journa Frontiers in Energy Research

Received: 18 January 2020 Accepted: 23 April 2020 Published: 21 May 2020

Citation: Li Y, Wang C and Li G (2020) A Mini-Review on High-Penetration Renewable Integration Into a Smarter

Grid. Front. Energy Res. 8:84 doi: 10.3389/fenrg.2020.00084 basic concepts, principles, as well as the presentation of the main applications of these technologies in smart grids. Additionally, the challenges faced and future prospects of these technologies are also discussed. In this paper, an attempt has been made to present a comprehensive review of the research on emerging technologies in the field of renewable integration in recent years.

Keywords: renewable integration, smart grid, smart inverter, renewable forecasting, IEC 61850, machine learning

\section{INTRODUCTION}

With increasing concerns on energy crisis and environmental pollution, clean and low-carbon energy transition has become a broad consensus around the world (Flore et al., 2019). Renewable generations such as wind power and photovoltaic (PV) have been undergoing booming development ( $\mathrm{Li}$ et al., 2017). However, with the increasing penetration of renewable energy resources, the issues associated with renewable generations have become increasingly prominent due to their inherent uncertainty and intermittence (Li et al., 2019a). In this context, emerging technologies such as smart inverters and machine learning (ML) have provided more regulatory means for better integration of high-penetration renewable energy. How to comprehensively leverage these emerging technologies for promoting the energy transition has great practical significance for today's power systems.

\section{AN OVERVIEW OF EMERGING TECHNOLOGIES}

This paper discusses the typical emerging technologies and their applications in smart grids, which have significant potentials to enable power grids to become smarter with high-penetration 
renewable integration (HPRI), shaping the future of electric power systems. Furthermore, this section also investigates the recent findings and critical knowledge to better understand and leverage these technologies in smart grids.

\section{Smart Inverters}

Smart inverters play a crucial role in ensuring the secure, reliable and efficient operation of an entire PV or WT gridconnected system. Besides DC-AC conversion, the inverters have functions such as circuit disconnection, charging of batteries, and maximum power point tracking (MPPT) (Ustun et al., 2019).

Smart inverters have some typical characteristics, such as plug and play, self-awareness, adaptability, autonomy, and cooperativeness (Arbab-Zavar et al., 2019), as shown in Figure 1.

The performance indicators are described as follows: (1) Plugand-play: A smart inverter needs to be compatible with standard communication protocols in power grids. (2) Self-awareness: The inverter can discover the parts that may be faulty, and then determine their exact locations, find out the cause of the faults, and be able to isolate them. (3) Adaptability: The inverter must be able to adjust itself to the changes in the system. (4) Autonomy: The inverter must be able to automatically switch operating modes according to operating conditions. (5) Cooperativeness: Inverters must be able to cooperate with other components in the grid.

In recent years, smart inverters have been widely used in various fields. Jha et al. (2019) presents a bi-level Volt-VAR optimization method which coordinates smart inverters and legacy voltage control devices for the purpose of reducing the customer power demand. A new smart inverter control as PVSTSTCOM for controlling the steady-state voltage and temporary overvoltage caused by unsymmetrical faults is proposed in Varma and Siavashi (2018). Almasalma et al. (2019) proposes a grid voltage support algorithm suitable for smart PV inverters. Mahmud et al. (2017) proposes a method for coordinating the operation of PV smart inverter control schemes with energy storage management systems for grid-connected PV system voltage regulation.

\section{Advanced Communication Protocols in Smart Grids}

A communication protocol refers to a rule system that allows entities of a communication system to transmit information. The protocol defines the rules, syntax, semantics, and synchronization of communication and error recovery approaches. The international standard IEC 61850 communication protocol is the first complete communication standard for smart substation automation systems, which has the following technical characteristics:

(1) System layering technology. IEC 61850 specifies the threelayer structure of the substation automation system: station layer, bay layer, and process layer.

(2) Object-oriented modeling technology. IEC 61850 adopts object-oriented technology, establishes a unified device and system model, and uses the XML-based safety check list (SCL) substation equipment communication configuration language.

(3) Abstract service communication interface (ASCI) technology. In order to realize a seamless communication network, ASCI can fully adapt to various communication protocols.

IEC 61850 has been successfully applied to many engineering fields such as substation automation (Moussa et al., 2016). IEC 61850-7-410 is a monitoring and communication standard for hydropower plants, IEC 61850-7-420 is a distributed energy monitoring standard which supports monitoring of a variety of distributed energies (Ustun et al., 2012), and IEC 61400-25 is a wind farm monitoring standard (Ahmed and Kim, 2014). These communication protocols have become an essential part of smart grids, which will play an increasingly important role in further power systems.

\section{Interoperability of Renewable Devices and Systems}

The interoperability refers to the ability which realizes secure, seamless, and transparent information exchange and data sharing through standard interfaces of a series of equipment and systems (Kiljander et al., 2014; Zhang et al., 2019).

In order to implement various interactions and transactions, smart grids must meet interoperability requirements (Ma et al., 2013). Interoperability is an important basis for participating in the formulation of international standards, such as IEC 61850 and IEEE P2030, which stipulates technical terms, evaluation criteria and applications of engineering principles for smart grid interoperability in the energy and information technology, and some network standards like transmission control protocol. Higher layers of interoperability are essential to achieve smart functions; and furthermore, the interoperability between renewable energy and systems is conducive to the HPRI.

\section{Peer-to-Peer Energy Trading}

Peer-to-peer (P2P) energy trading is an important market mechanism in which users can directly conduct transactions with others. This mechanism can optimize the energy structure and promote local consumptions of distributed energies.

The research on P2P energy trading has recently become a hot topic. Li et al. (2018) presents a framework for transactive energy trading in distribution systems. In a P2P energy sharing network, prosumers with surplus energy can directly transmit energy to others with insufficient supply through transmission lines (Morstyn et al., 2018). In addition, a good trading scheme should enable every prosumer to benefit from a P2P energy network. In recent years, blockchain technology has been widely studied in the field of energy trading due to its decentralization, anonymity, and high reliability. Most simulations of P2P energy trading are based on game theory (Paudel et al., 2018) since the theory is beneficial to balance the interests of electricity suppliers and consumers in a local energy market. 


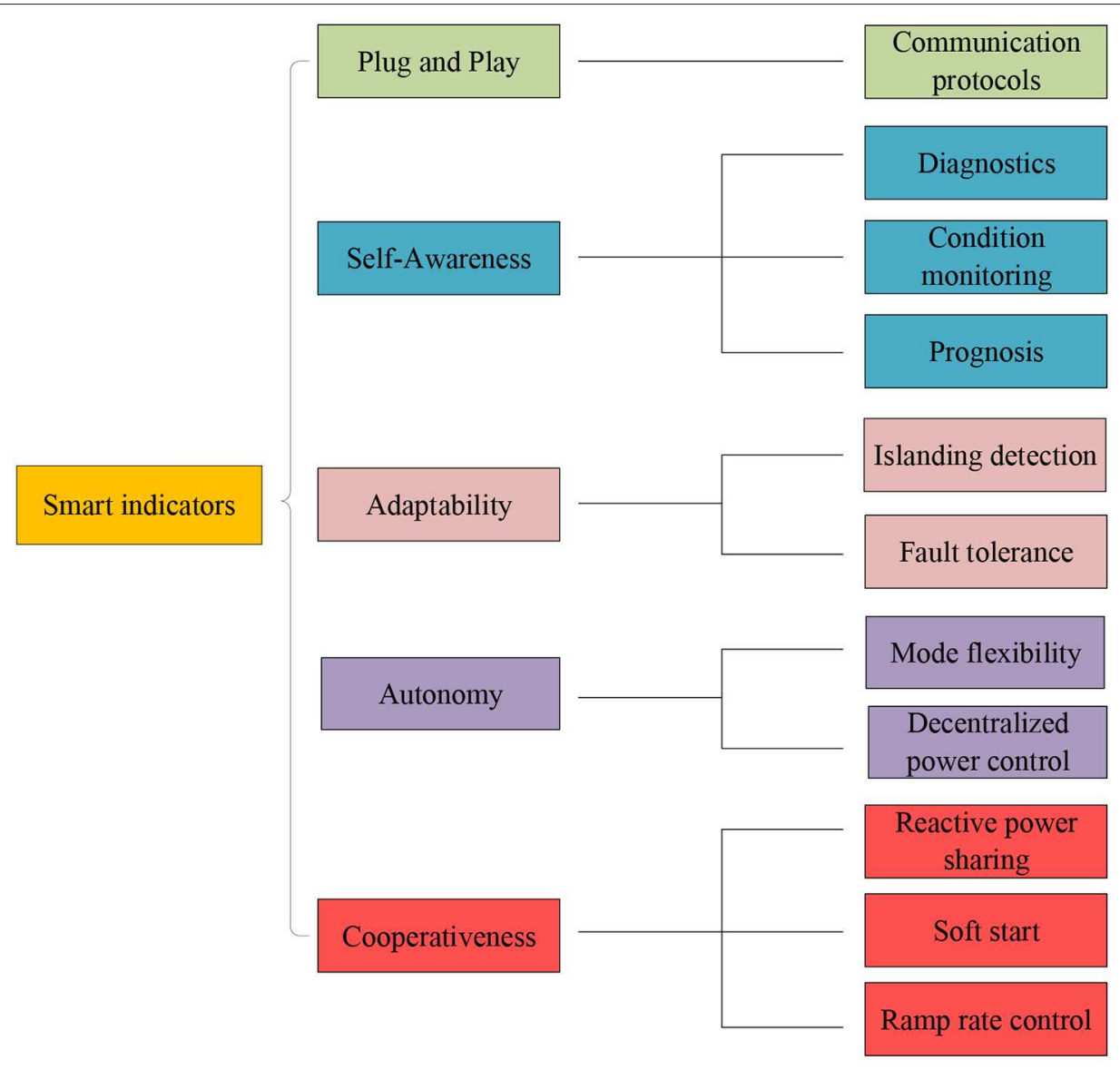

FIGURE 1 | Smart indicators of smart inverters.

\section{Renewable Forecasting}

With the development of renewable generations, their uncertainty and intermittence pose great challenges to the safe operation of power systems (Li et al., 2019b; Wang et al., 2019). Renewable forecasting has always been regarded as a crucial technique for addressing these challenges, and it can be classified as different classes according to different criteria. In terms of spatial scales, they can be divided into singlestation forecasting and regional forecasting; regarding temporal scales, they are separated into ultra-short-term forecasting $(0 \sim 4 \mathrm{~h})$, short-term forecasting $(0-72 \mathrm{~h})$, and medium-longterm forecasting (1 month-1 year) and long-term forecasting (>1 year).

In recent years, various renewable forecasting methods have been presented. An adaptive diffusion kernel density estimation-based probabilistic optimal power flow calculation method is proposed in Li G. et al. (2019). Andrade and Bessa (2017) enhances the renewable prediction performance via the information from a numerical weather forecast grid. An improved aggregated probability wind power prediction framework based on spatio-temporal correlation is proposed in Sun et al. (2019). Gao et al. (2019) presents a time-series prediction model under ideal and non-ideal weather conditions for day-ahead PV power outputs.

\section{Machine Learning}

Machine learning (ML) is a powerful technique that analyzes and learns a large amount of existing or generated data for predictions or decisions (Pallonetto et al., 2019). It belongs to the scientific study of algorithms and statistical models that computers use to perform a specific task without using explicit instructions. ML algorithms build a mathematical model by using sample data sets, namely knowledge base, to make a prediction (classification or regression) without being explicitly programmed to perform the task.

In general, machine learning can be divided into four categories: supervised learning, unsupervised learning, semisupervised learning, and reinforcement learning. This section summarizes the application of these learning algorithms in smart grids, which is shown in Table 1.

\section{Other Technologies}

Besides the above six emerging technologies, there are many emerging important technologies about smart grid and HPRI are developed in recent years. An integrated energy system (IES), which comprehensively utilizes multiple energies in a region to achieve coordinated planning and operations among multiple energy forms, is able to provide more regulatory means available for enabling greater consumption of renewable 
TABLE 1 | Summary of application of ML algorithms in smart grids.

\begin{tabular}{|c|c|c|c|}
\hline ML algorithms & Definitions & Typical algorithms & Application scenarios \\
\hline Supervised learning & $\begin{array}{l}\text { A ML approach that learns a function via } \\
\text { example input-output pairs. }\end{array}$ & $\begin{array}{l}\text { Logistic regression; } \\
\text { naive Bayesian classifier; } \\
\text { decision trees; } \\
\text { K-nearest neighbor algorithm. }\end{array}$ & $\begin{array}{l}\text { Substation clustering and classification; } \\
\text { Security and stability assessment (Li and Yang, } \\
\text { 2017); } \\
\text { electricity price and load forecasting (Ghasemi } \\
\text { et al., 2016). }\end{array}$ \\
\hline Unsupervised learning & $\begin{array}{l}\text { A self-organized learning which helps find } \\
\text { unknown patterns in knowledge base } \\
\text { without pre-given labels }\end{array}$ & $\begin{array}{l}\text { K-means clustering; self-organizing map; } \\
\text { autoencoders; } \\
\text { generative adversarial networks. }\end{array}$ & $\begin{array}{l}\text { Clustering analysis of typical days (Chen et al., } \\
\text { 2019); Cascading analysis of power grids (Yan } \\
\text { et al., 2013). }\end{array}$ \\
\hline Semi-supervised learning & $\begin{array}{l}\text { A ML approach that combines labeled and } \\
\text { unlabeled data during training }\end{array}$ & $\begin{array}{l}\text { Self-training algorithm; co-training } \\
\text { algorithm; graph-based method. }\end{array}$ & $\begin{array}{l}\text { Non-intrusive load monitoring; non-technical } \\
\text { loss detection in the smart grid. }\end{array}$ \\
\hline Reinforcement learning & $\begin{array}{l}\text { A ML approach concerned with how to } \\
\text { maximize reward by taking actions }\end{array}$ & $\begin{array}{l}\text { Q-learning; } \\
\text { temporal difference learning; } \\
\text { Monte Carlo learning. }\end{array}$ & $\begin{array}{l}\text { Dynamic pricing and energy consumption } \\
\text { scheduling (Kim et al., 2015); supply-demand } \\
\text { Stackelberg game of smart grid. }\end{array}$ \\
\hline
\end{tabular}

energies. VSC-HVDC technology is another means to promote renewable energy consumption. In addition, improving flexibility through leveraging multiple resources such as demand response, energy storage and thermal inertia of buildings, is an important technology to cope with the variation and uncertainty of the high penetration of renewable energy in planning and operation process (Li et al., 2020).

\section{CHALLENGES AND FUTURE TRENDS}

\section{Challenges}

Although the mentioned representative emerging technologies have played significant roles in the integration of highpenetration renewable energies, they still face some challenges. The following are some examples:

(1) In terms of a smart inverter, no matter how well it is designed, it still has some disadvantages such as time delay. Therefore, the development of control structures that can effectively reduce communication requirements is an interesting topic for future research.

(2) For P2P technology, due to the communication requirement in energy trading, future work will focus on the scalable architecture of communication systems to enable them to provide low latency and support compatibility with information and communication technology systems with different versions.

(3) Regarding machine learning, most of the existing $\mathrm{ML}$ techniques act like "black boxes" with poor transparency, which makes it difficult to deploy them in some highrisk scenarios. In addition, the issues on data security and privacy-preserving limits to a certain extent its popularization in practical applications.

\section{Future Trends}

The researchers and engineers in academia and industry need to find solutions to address the above-mentioned problems from the following aspects: (1) First of all, new emerging technologies that are beneficial to renewable integration should be studied extensively and intensively for maximizing their potential. (2) What's more, sophisticated approaches to technology integration deserves more attention to advance the study in this field. (3) Last but not least, the application of the emerging technologies related to renewable integration into the electric power industry should be encouraged and strengthened. Specifically speaking, future smart inverters are developing toward higher efficiency and smaller size; the application ranges of interoperability and communication protocols are supposed to be expanded; regarding $M L$, deep integration of ML methods to make them more effective, intelligent, and interprein the application of HPRI should be further studied. By combining the advanced technologies with practical applications, the industrialization of technology will be promoted for renewable integration into smarter grids.

\section{CONCLUSIONS}

Facing increasingly serious energy crisis and environmental pollution, today's power system is undergoing profound changes and evolves toward a smarter grid. In this context, some new emerging technologies come into being and provide more regulatory means available for contributing to renewable integration. This paper cover focused aspects of HPRI and its recent developments. This work firstly presents a critical minireview of the concept and application of these technologies for reshaping future power grids with high-penetration renewables. In addition, the challenges and potential future developments in the field are also discussed.

\section{AUTHOR CONTRIBUTIONS}

YL contributed conception and design of the study. CW wrote the first draft of the manuscript. YL and GL are in charge of project administration. All authors contributed to manuscript revision, read and approved the submitted version.

\section{FUNDING}

This work was partly supported by the State Grid Corporation of China under Grant No. 5102-201999343A-0-0-00. 


\section{REFERENCES}

Ahmed, M., and Kim, Y. C. (2014). Hierarchical communication network architectures for offshore wind power farms. Energies 7, 3420-3437. doi: $10.3390 /$ en7053420

Almasalma, H., Claeys, S., and Deconinck, G. (2019). Peer-to-peer-based integrated grid voltage support function for smart photovoltaic inverters. Appl. Energy 239, 1037-1048. doi: 10.1016/j.apenergy.2019.01.249

Andrade, J. R., and Bessa, R. J. (2017). Improving renewable energy forecasting with a grid of numerical weather predictions. IEEE Trans. Sustain. Energy 8, 1571-1580. doi: 10.1109/TSTE.2017.2694340

Arbab-Zavar, B., Palacios-Garcia, E. J., Vasquez, J. C., and Guerrero, J. M. (2019). Smart inverters for microgrid applications: A Review. Energies 12:840. doi: $10.3390 /$ en 12050840

Chen, Q., Xia, M., Zhou, Y., Cai, H., Wu, J., and Zhang, H. (2019). Optimal planning for partially self-sufficient microgrid with limited annual electricity exchange with distribution grid. IEEE Access 7, 123505-123520. doi: 10.1109/ACCESS.2019.2936762

Flore, A., Clausen, M., and Uslar, M. (2019). Migration paths for smart grids and their evaluation using a case study. Front. Energy Res. 7:134. doi: $10.3389 /$ fenrg. 2019.00134

Gao, M., Li, J., Hong, F., and Long, D. (2019). Day-ahead power forecasting in a large-scale photovoltaic plant based on weather classification using LSTM. Energy 187:115838. doi: 10.1016/j.energy.2019.07.168

Ghasemi, A., Shayeghi, H., Moradzadeh, M., and Nooshyar, M. (2016). A novel hybrid algorithm for electricity price and load forecasting in smart grids with demand-side management. Appl. Energy 177, 40-59. doi: 10.1016/j.apenergy.2016.05.083

Jha, R. R., Dubey, A., Liu, C. C., and Schneider, K. P. (2019). Bi-Level Volt-VAR optimization to coordinate smart inverters with voltage control devices. IEEE Trans. Power Syst. 34, 1801-1813. doi: 10.1109/TPWRS.2018.2890613

Kiljander, J., D’elia, A., Morandi, F., Hyttinen, P., Takalo-Mattila, J., Ylisaukko-Oja, A., et al. (2014). Semantic interoperability architecture for pervasive computing and internet of things. IEEE Access 2, 856-873. doi: 10.1109/ACCESS.2014.2347992

Kim, B. G., Zhang, Y., Van Der Schaar, M., and Lee, J. W. (2015). Dynamic pricing and energy consumption scheduling with reinforcement learning. IEEE Trans. Smart Grid 7, 2187-2198. doi: 10.1109/TSG.2015.2495145

Li, G., Lu, W., Bian, J., Qin, F., and Wu, J. (2019). Probabilistic optimal power flow calculation method based on adaptive diffusion kernel density estimation. Front. Energy Res. 7:128. doi: 10.3389/fenrg.2019.00128

Li, J., Zhang, C., Xu, Z., Wang, J., Zhao, J., Zhang, Y., et al. (2018). Distributed transactive energy trading framework in distribution networks. IEEE Trans. Power Syst. 33, 7215-7227. doi: 10.1109/TPWRS.2018.2854649

Li, Y., Wang, C., Li, G., Wang, J., Zhao, D., and Chen, C., (2020). Improving operational flexibility of integrated energy system with uncertain renewable generations considering thermal inertia of buildings. Energy Convers. Manage. 207:112526. doi: 10.1016/j.enconman.2020.112526

Li, Y., Wang, P., Gooi, H. B., Ye, J., and Wu, L. (2017). Multi-objective optimal dispatch of microgrid under uncertainties via interval optimization. IEEE Trans. Smart Grid 10, 2046-2058. doi: 10.1109/TSG.2017.2787790

Li, Y., and Yang, Z. (2017). Application of EOS-ELM with binary Jaya-based feature selection to real-time transient stability assessment using PMU data. IEEE Access. 5, 23092-23101. doi: 10.1109/ACCESS.2017.2765626

Li, Y., Yang, Z., Li, G., Zhao, D., and Tian, W. (2019a). Optimal scheduling of an isolated microgrid with battery storage considering load and renewable generation uncertainties. IEEE Trans. Indust. Electron. 66, 1565-1575. doi: 10.1109/TIE.2018.2840498
Li, Y., Yang, Z., Zhao, D., Lei, H., Cui, B., and Li, S. (2019b). Incorporating energy storage and user experience in isolated microgrid dispatch using a multi-objective model. IET Renew. Power Gen. 13, 973-981. doi: 10.1049 /iet-rpg.2018.5862

Ma, R., Chen, H. H., Huang, Y. R., and Meng, W. (2013). Smart grid communication: Its challenges and opportunities. IEEE Trans. Smart Grid 4, 36-46. doi: 10.1109/TSG.2012.2225851

Mahmud, N., Zahedi, A., and Mahmud, A. (2017). A cooperative operation of novel PV inverter control scheme and storage energy management system based on ANFIS for voltage regulation of grid-tied PV system. IEEE Trans. Industr. Informat. 13, 2657-2668. doi: 10.1109/TII.2017.2651111

Morstyn, T., Farrell, N., Darby, S. J., and McCulloch, M. D. (2018). Using peerto-peer energy-trading platforms to incentivize prosumers to form federated power plants. Nat. Energy 3, 94-101. doi: 10.1038/s41560-017-0075-y

Moussa, B., Debbabi, M., and Assi, C. (2016). A detection and mitigation model for PTP delay attack in an IEC 61850 substation. IEEE Trans. Smart Grid 9, 3954-3965. doi: 10.1109/TSG.2016.2644618

Pallonetto, F., De Rosa, M., Milano, F., and Finn, D. P. (2019). Demand response algorithms for smart-grid ready residential buildings using machine learning models. Appl. Energy 239, 1265-1282. doi: 10.1016/j.apenergy.2019.02.020

Paudel, A., Chaudhari, K., Long, C., and Gooi, H. B. (2018). Peer-to-peer energy trading in a prosumer-based community microgrid: a game-theoretic model. IEEE Trans. Industr. Electron. 66, 6087-6097. doi: 10.1109/TIE.2018.2874578

Sun, M., Feng, C., and Zhang, J. (2019). Conditional aggregated probabilistic wind power forecasting based on spatio-temporal correlation. Appl. Energy 256:113842. doi: 10.1016/j.apenergy.2019.113842

Ustun, T. S., Hashimoto, J., and Otani, K. (2019). Impact of smart inverters on feeder hosting capacity of distribution networks. IEEE Access 7, 163526-163536. doi: 10.1109/ACCESS.2019.2952569

Ustun, T. S., Ozansoy, C., and Zayegh, A. (2012). Modeling of a centralized microgrid protection system and distributed energy resources according to IEC 61850-7-420. IEEE Trans. Syst. 27, 1560-1567. doi: 10.1109/TPWRS.2012.2185072

Varma, R. K., and Siavashi, E. M. (2018). Enhancement of solar farm connectivity with smart PV inverter PV-STATCOM. IEEE Trans. Sustain. Energy 10, 1161-1171. doi: 10.1109/TSTE.2018.2862405

Wang, Z., Kang, J., Cheng, L., Pei, Z., Dong, C., and Liang, Z. (2019). Mixed Gaussian models for modelling fluctuation process characteristics of photovoltaic outputs. Front. Energy Res. 7:76. doi: 10.3389/fenrg.2019. 00076

Yan, J., Zhu, Y., He, H., and Sun, Y. (2013). Multi-contingency cascading analysis of smart grid based on self-organizing map. IEEE Trans. Informat. Forensics Sec. 8, 646-656. doi: 10.1109/TIFS.2013.2249065

Zhang, Y., Yan, Z., Kan, T., and Mi, C. (2019). Interoperability study of fast wireless charging and normal wireless charging of electric vehicles with a shared receiver. IET Power Electron. 12, 2551-2558. doi: 10.1049/iet-pel. 2018.6080

Conflict of Interest: The authors declare that the research was conducted in the absence of any commercial or financial relationships that could be construed as a potential conflict of interest.

Copyright (c) $2020 \mathrm{Li}$, Wang and Li. This is an open-access article distributed under the terms of the Creative Commons Attribution License (CC BY). The use, distribution or reproduction in other forums is permitted, provided the original author(s) and the copyright owner(s) are credited and that the original publication in this journal is cited, in accordance with accepted academic practice. No use, distribution or reproduction is permitted which does not comply with these terms. 